\title{
Farmaconutrición: ¿El fin de una era?
}

\author{
Pharmaconutrition: The End of an Era?
}

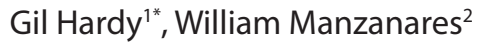 \\ Recibido: 1 de noviembre de 2018. Aceptado para publicación: 1 de diciembre de 2018 \\ Publicado en línea, diciembre 5 de 2018 \\ https://doi.org/10.35454/rncm.v2n1.057
}

\section{Resumen}

La farmaconutrición ha sido un área interesante de investigación en cuidados intensivos. Al inicio, los ensayos con dosis altas de glutamina o selenio informaron resultados alentadores, pero a menudo no tuvieron el poder estadístico suficiente. No obstante, las revisiones sistemáticas y los meta-análisis realizados hasta 2015 encontraron reducciones significativas en las tasas de infección y/o mortalidad de los enfermos en estado crítico. Sin embargo, los meta-análisis posteriores no han mostrado mejoría en los resultados clínicos. En consecuencia, las directrices actuales no hacen ninguna recomendación para el tratamiento con altas dosis de nutrientes. ¿Es éste el fin de la era de la farmaconutrición? No necesariamente! Es hora de volver a lo básico y adoptar un enfoque más farmacéutico clasificando los farmaconutrientes como medicamentos; establecer mejor su estabilidad, farmacología, toxicología y seguridad in vitro e in vivo, y luego determinar las interacciones fármaco-fármaco o fármaco-nutriente antes de proceder a los estudios de farmacocinética y farmacodinámica. Además, debemos investigar la verdadera naturaleza de la deficiencia de nutrientes en las personas gravemente enfermas. ¿Cuál es la diferencia entre una deficiencia en una población con cifras de Selenio naturalmente bajas y una población con cifras "normales"? ¿Qué sucede con una dosis alta de un dipéptido de glutamina sintética en un paciente con deficiencia de nutrientes, o con insuficiencia orgánica, y qué sucede con el exceso de farmaconutriente que no es utilizado por un paciente en buen estado nutricional?

Estas preguntas básicas no fueron investigadas en forma adecuada en el pasado, donde se administraron dosis suprafisiológicas fijas a todos los pacientes, de los cuales una proporción significativa no tenía deficiencia de nutrientes. Cuando se han generado datos de calidad sobre estos parámetros preclínicos, podemos determinar con mayor precisión las indicaciones, la posología óptima y las mejores guías para nuevas investigaciones clínicas e iniciar una nueva era de la Nutrición Farmacéutica.

Palabras clave: farmaconutrición, glutamina/dipéptidos de glutamina, selenio, cuidados intensivos.

\section{Summary}

Pharmaconutrition has been an interesting area of research in critical care. Initially, trials with high dose glutamine and/or selenium reported promising outcomes but were often underpowered. Notwithstanding, systematic reviews and meta-analyses conducted up to 2015 found significant reductions in infection and/ or mortality rates in the critically ill. However, later meta- analyses have not shown improvement in clinical outcomes. Consequently, current guidelines do not make any recommendations for high dose nutrient therapy. Is this the end of the pharmaconutrition era? Not necessarily! It is time to return to basics and adopt a more pharmaceutical approach by categorising pharmaconutrients as drugs; better establish their in vitro and in vivo stability, pharmacology, toxicology and safety, then determine any drug-drug or drug-nutrient interactions before proceeding to pharmacokinetics and pharmacodynamics studies. We must additionally investigate the true nature of nutrient deficiency in the critically ill. How different is a deficiency in a naturally low Selenium population versus a 'normal' population? What happens to a high dose of a synthetic glutamine dipeptide in a nutrient deficient patient, or one with organ failure, and what happens to the excess pharmaconutrient that is not utilised by a nutritionally replete patient?

These basic questions were inadequately investigated in the past, where fixed supraphysiological doses were administered to all patients, a significant proportion of whom were not nutrient deficient. When quality data have been generated on these preclinical parameters, we can more accurately determine indications, optimum posology and better guidelines for new clinical investigations and begin a new era of Pharmaceutical Nutrition.

Keywords: Pharmaconutrition; Glutamine dipeptides; Selenium, Critical care.

Profesor Emérito de Nutrición Clínica, Auckland Nueva Zelanda

Profesor Agregado de Medicina Intensiva, Cátedra de Medicina Intensiva. Facultad de Medicina, Universidad de la República, Montevideo, Uruguay

*Correspondencia: Gil Hardy gil.hardy50@gmail.com 


\section{INTRODUCCIÓN}

La glutamina (GLN), la arginina (ARG) y el selenio (Se) administrados como tratamiento farmacológico de altas dosis han sido tema de investigación nutricional en las últimas tres décadas. Muchos ensayos controlados aleatorios (ECA) pequeños, de un solo centro, informaron resultados prometedores, pero a menudo tuvieron poco poder estadístico ${ }^{(1)}$. No obstante, la mayoría de las revisiones y meta-análisis sistemáticos realizados hasta 2015 encontraron reducciones significativas en las tasas de infección y mortalidad cuando se administraron altas dosis de GLN como dipéptido alanil-L-glutamina (DIPEP) y Se (como selenita) a los enfermos en estado crítico $^{(2)}$. Sin embargo, después de la publicación de un ECA más grande y negativo ${ }^{(3-5)}$, los meta-análisis posteriores concluyeron que las altas dosis de Se o GLN, no mejoran los resultados clínicos en la atención crítica ${ }^{(6)}$. En consecuencia, las pruebas actuales no parecen apoyar el concepto de farmaconutrición y las directrices clínicas de las principales sociedades profesionales no recomiendan el tratamiento con altas dosis de nutrientes ${ }^{(7,8)}$.

Entonces, ¿es éste el fin de la era de la farmaconutrición en los enfermos en estado crítico? NO NECESARIAMENTE!

\section{ANTECEDENTES}

El estado fisiológico inmunológico del cuerpo favorece la descomposición de las proteínas estructurales en lugar de un mayor uso de fuentes exógenas de nitrógeno. La L-glutamina (GLN), considerada "condicionalmente esencial”, es el aminoácido (AA) más abundante en el torrente sanguíneo. La GLN es un sustrato respiratorio clave para la rápida división de células como los enterocitos y las células inmunitarias, aumentando su actividad fagocítica. También es un precursor de la arginina a través de la vía citrulino-arginina. En la sepsis, los niveles de AA en plasma disminuyen debido a la síntesis de proteínas relacionadas con el sistema inmunológico y a la producción de glucosa a través de la gluconeogénesis en el hígado. Los niveles plasmáticos de ARG disminuyen a medida que el AA se utiliza para la conversión en óxido nítrico $(\mathrm{ON})$ y para la participación en el ciclo de la urea para desechar el amoníaco liberado por el metabolismo de otros AA.

Los aminoácidos azufrados (AAz) y sus metabolitos principales tienen un papel fundamental en los sistemas antioxidantes celulares y atenúan los síntomas proinflamatorios. En una investigación muy interesante presentada en la Sociedad de Nutrición Parenteral y
Enteral de Asia PENSA 2018, Kwang Suk Ko en la Universidad de Ewha Womans, Corea ${ }^{(9)}$ añadió AAz en macrófagos deficientes en prohibitin-1 seguido de la activación de lipopolisacáridos (LPS). La prohibitin-1 (una chaperona mitocondrial y un gen supresor de tumores hepáticos) agravó la respuesta inflamatoria de los macrófagos, mientras que los AAz atenuaron esos efectos adversos en comparación con los controles. Como la GLN y los AAz son sustratos para el principal antioxidante del cuerpo, el glutatión (GSH), estos datos proporcionan una evidencia creciente de que la suplementación podría ser útil para los pacientes sépticos a través de esta relación sinérgica que necesita mayor exploración.

\section{GLUTAMINA E INFLAMACIÓN}

La GLN es importante para el metabolismo intestinal, en especial después del estrés, y las dietas con GLN mejoran la morfología y la función intestinal. Como resultado, la GLN ha sido estudiada durante mucho tiempo como un agente prometedor para preservar la función intestinal y la recuperación durante la lesión o el estrés. Aunque el mecanismo por el cual la GLN ejerce sus efectos beneficiosos no se comprende completamente, parece estar correlacionado con la mejora de la función de barrera intestinal, la lesión oxidante y la inhibición de los procesos inflamatorios, como la activación de NF- $\mathrm{kB}$ y la producción de TNF-a. Muchos investigadores han reportado que la terapia con GLN mejora los resultados de los modelos experimentales de colitis, incluyendo la investigación de Hern Ku Lee y sus colegas de la Facultad de Medicina de la Universidad Nacional de Chonbuk, Corea, que proporciona evidencia de que la GLN puede atenuar enfermedades inflamatorias como la sepsis, el asma, la anafilaxia tardía, la dermatitis y la colitis en ratones a una dosis de $20 \mathrm{mg} /$ ratón $^{(10)}$. Han demostrado que la GLN aumenta la actividad de la quinasa extracelular regulada por señal (ERK) MAPK mediante la activación de la vía que involucra Ca2+/Ras/c-Raf/MEK (cascada ERK). La ERK fosforila la protein fosfatasa MAPK fosfatasa-1(MKP-1) en dos residuos de serina carboxil-terminal - serina 359 y serina 364, lo que mejora la estabilización de MKP-1, lo que da como resultado la inducción temprana de MKP-1 para desactivar el cPLA2, ya sea mediante la desfosforilación de la proteína quinasa activada por mitógeno p38 (MAPK), una importante vía de entrada para la fosforilación de cPLA2, o bien mediante la desfosforilación directa de cPLA2, debido a la mejora de la interacción física entre los procesos 
de fosforilación inducida por la GLN, el MKP-1 y el cPLA2. Estos datos sugieren que la GLN funciona como un inhibidor endógeno de cPLA2 mediante la inducción de MKP-1, que a través de la reactivación de p38 y cPLA2 resulta en la supresión de muchos mediadores inflamatorios importantes, incluyendo especies reactivas de oxígeno.

\section{DEFICIENCIA DE GLUTAMINA O HIPOGLUTAMINEMIA}

El pionero de la GLN, el profesor Jan Wernerman (Hospital Universitario del Karolinska, Suecia) ha acuñado el término "hipoglutaminemia" en lugar de "deficiencia" para definir mejor los niveles reducidos de GLN en los enfermos en estado crítico, durante los períodos de inflamación sistémica y sepsis (valor normal de GLN en plasma: 420 - $930 \mu \mathrm{mol} / \mathrm{L}$ ). La hipoglutaminemia, su prevalencia estimada se encuentra entre $31 \%$ y $65 \%$ de los pacientes ingresados en la $\mathrm{UCI}^{(11)}$, tiene implicaciones importantes. Ciertas condiciones y síntomas clínicos adversos se asocian con mayor frecuencia a niveles bajos de GLN en plasma y pueden considerarse, potencialmente, indicadores clínicos de deficiencia. Estos incluyen la gravedad de la enfermedad (puntuaciones APACHE II), la presencia de infecciones (medidas por PCR, IL-6, entre otros), la edad avanzada, los niveles más bajos de albúmina y los ingresos no electivos en la UCI. Los niveles de GLN son significativamente más bajos en pacientes con infección $(\mathrm{p}=0,01)^{(11)}$. Los bajos niveles de GLN en plasma se han correlacionado con una mayor duración de la estancia hospitalaria (LOS) y en las UCI, así como con una mejoría en la mortalidad a los 6 meses $^{(12,13)}$ y se han propuesto como un indicador pronóstico independiente de mortalidad ${ }^{(14)}$.

\section{SUPLEMENTO DE GLUTAMINA EN ENFERMOS EN ESTADO CRÍTICO}

Históricamente, el ensayo controlado aleatorio de un solo centro que mostró beneficios clínicos con la administración de suplemento de LGN, utilizó en especial soluciones preparadas, en forma aséptica, de L-GLN puro $^{(15)}$. Los meta-análisis iniciales de estos ensayos concluyeron que la administración de altas dosis de suplementos de GLN condujo a una reducción significativa de las infecciones hospitalarias, la mortalidad, la estancia en el hospital y en la UCI. Aunque el estudio escandinavo ${ }^{(16)}$ informó una disminución de la mortalidad durante la estancia en la UCI, no se mantuvo a los seis meses. Sin embargo, en el momento del alta, la hipoglutaminemia no fue muy prevalente y no fue un factor de predicción de resultados desfavorables. En contraste, las primeras investigaciones de Griffiths, et al. observaron una disminución de la mortalidad a los 6 meses, pero es notable que la restauración de los niveles de GLN muscular duró más de 6 meses ${ }^{(17)}$. Esto plantea la pregunta de si sería ventajoso prolongar la terapia con GLN después de la estancia en la UCI.

Por el contrario, los altos niveles de GLN luego de la suplementación con DIPEP se han asociado a resultados adversos. Dos grandes ECA multicéntricos que evaluaron la eficacia de los cocteles antioxidantes, incluido el DIPEP más Selenio en pacientes ventilados en la UCI, no lograron reproducir esos resultados positivos iniciales. El ensayo multicéntrico REDOXS ${ }^{(3)}$, que incluyó a 1223 pacientes ventilados, asignados al azar, de $40 \mathrm{UCI}$, mostró un aumento de la mortalidad en los que recibieron altas dosis de DIPEP. El ensayo MetaPlus ${ }^{(4)}$ en 14 UCI, que asignó al azar a 301 pacientes de UCI ventilados a recibir nutrición enteral enriquecida con DIPEP versus una dieta isocalórica, observó un aumento de la mortalidad a los seis meses en el grupo suplementado con DIPEP. Entonces, ¿ por qué estas diferencias aparentes en el resultado entre los estudios iniciales y los posteriores?

Es evidente que hay necesidad de investigar más a fondo las diversas preguntas que aún permanecen sin respuesta, pero antes de comenzar ensayos clínicos mejor diseñados es el momento de volver a lo básico y adoptar un enfoque más farmacéutico. Los farmaconutrientes sintéticos como los dipéptidos deben clasificarse e investigarse como fármacos. Es esencial establecer la estabilidad in vitro e in vivo de estos farmaconutrientes, su farmacología, toxicología y seguridad a partir de modelos animales, el método preferido de administración y las curvas de respuesta del medicamento a dosis muy altas. También deben determinarse las interacciones fármaco-fármaco o fármaco-nutriente durante el uso antes de proceder a estudios de farmacocinética humana y farmacodinámica.

En retrospectiva, estos protocolos farmacéuticos y metabólicos básicos no fueron investigados en forma adecuada antes de iniciar estudios clínicos como los ensayos REDOXS, Metaplus o SISPCT ${ }^{(3-5)}$. El ensayo REDOXS también tuvo un problema de aleatorización, en el que se administraron dosis fijas suprafisiológicas de DIPEP a todos los pacientes, independiente de su peso, edad o sexo, una proporción significativa de los cuales no tenían hipoglutaminemia (ni eran necesariamente "hiposelenémicos"). El régimen de apoyo nutricional también fue inadecuado: la ingesta de energía de la mayoría de 
los pacientes fue muy baja, el grupo de estudio recibió mucha más proteína de una mezcla desequilibrada de AA, que el hígado ya comprometido de estos pacientes tuvieron que oxidar, mientras que la ingesta de nitrógeno fue mucho menor en los controles. Todavía no está claro la causa del resultado desfavorable para los pacientes en el grupo suplementado con DIPEP, pero el subgrupo de pacientes norteamericanos, para quienes se disponía de concentraciones plasmáticas de GLN, mostró que las altas concentraciones plasmáticas no eran la razón. Cabe destacar que tanto la GLN como la alanina (ALA) están implicados en la gluconeogénesis, pero hasta la fecha nadie ha informado o comentado sobre los niveles igualmente altos de ALA resultantes de la administración de suplemento de DIPEP.

Dado que las principales sociedades de nutrición clínica recomiendan en la actualidad no administrar suplementos rutinarios en altas dosis hasta cuando se disponga de datos adicionales, se necesitan ensayos multicéntricos más grandes y mejor diseñados. Werneman señala que la hipótesis de que la suplementación con GLN podría ser beneficiosa para los pacientes con hipoglutaminemia en el momento del ingreso en la UCI nunca se ha probado de manera adecuada. A pesar de una multitud de ensayos clínicos, ningún estudio ha verificado si la hipoglutaminemia era prevalente o no en el ingreso y ningún estudio ha verificado que la suplementación pueda convertir la hipoglutaminemia en normoglutaminemia. En los pacientes de larga estancia en la UCI, el flujo de GLN del tejido muscular sigue siendo alto, pero este proceso metabólico parece no estar inhibido por la GLN exógena. Una mayor concentración de GLN en plasma puede ser ventajosa para la utilización de GLN en el hígado y el intestino. Aunque la prevalencia reportada de GLN plasmática alta $(6,7 \%$ a $14 \%)$ es menor que la prevalencia de deficiencia, se está haciendo cada vez más evidente que la asociación de los niveles de GLN plasmática circulante con la mortalidad parece seguir una curva en forma de $\mathrm{U}^{(18)}$. El alto contenido de GLN parece ser más común en la enfermedad hepática y correlacionarse con el grado de insuficiencia hepática ${ }^{(16)}$.

En consecuencia, debemos establecer la verdadera naturaleza de las deficiencias/anemias de los farmaconutrientes, como la GLN o el Se, en los enfermos en estado crítico. ¿Cómo, dónde y por qué ocurre? ¿Los niveles plasmáticos y otros marcadores sustitutos reflejan una verdadera deficiencia o "anemia"? ¿Cuál es la diferencia entre una deficiencia de nutrientes en una población con niveles naturalmente bajos y una población "normal"? ¿Qué sucede exactamente con una dosis alta de DIPEP sintético en un paciente con deficiencia de nutrientes en estado crítico o con insuficiencia orgánica, y qué sucede con el exceso de farmaconutrientes que no es utilizado por un paciente en adecuado estado nutricional $?^{(19)}$

\section{EN RESUMEN}

Sin más pruebas de seguridad y eficacia, los farmaconutrientes en altas dosis no deben administrarse a pacientes que no están siendo alimentados en forma adecuada. Por otro lado, la suplementación en dosis "nutricionales" para pacientes con hipoglutaminemia puede ser beneficiosa sin ninguna preocupación de seguridad, cuando se administra como parte de un régimen de terapia nutricional completa. Hasta tanto llegue el momento en que las determinaciones de plasma se puedan realizar de forma precisa y rutinaria al lado de la cama, se debe prestar atención a la identificación de los pacientes en riesgo ${ }^{(10)}$ y los médicos deben confiar en los indicadores clínicos para identificar a esos individuos antes de que se implemente el tratamiento.

Cuando se hayan generado datos de calidad sobre todos estos parámetros preclínicos, podremos llevar a cabo investigaciones clínicas para confirmar la validez de las hipótesis, determinar con mayor precisión las indicaciones, la posología óptima y desarrollar mejores directrices para nuevas investigaciones clínicas sobre los beneficios potenciales del tratamiento con farmaconutrientes en altas dosis. Comprender las funciones y el metabolismo de cada uno de los farmaconutrientes podría significar un mejor pronóstico para los pacientes gravemente enfermos e inmunocomprometidos que están luchando contra organismos invasores que intentan alterar sus procesos inmunológicos normales.

Con toda certeza esto no es el fin de una era, sino el comienzo de una nueva y emocionante era, la "Era de la Nutrición Farmacéutica”.

\section{Financiación}

El presente estudio no tuvo financiación.

\section{Conflicto de intereses}

Los autores declaran no tener conflicto de intereses.

\section{Referencias bibliográficas}

1. Hardy G, Hardy IJ. Can glutamine enable the critically ill to cope better with infection? J Parenter Enteral Nutr JPEN. 2008;32:489-91. 
2. Wischmeyer P, Dhaliwal R, McCall M, Ziegler TR, Heyland DK. Parenteral glutamine supplementation in critical illness: a systematic review. Crit Care. 2014;18: R76.

3. Heyland D, Muscedere J, Wischmeyer PE, Cook D, Jones G, Albert $\mathrm{M}$, et al. A randomized trial of glutamine and antioxidants in critically ill patients. N Engl J Med. 2013;368:1489-97.

4. van Zanten A, Sztark F, Kaisers UX, Zielmann S, Felbinger TW, Sablotzki AR, et al. High-protein enteral nutrition enriched with immune-modulating nutrients vs standard highprotein enteral nutrition and nosocomial infections in the ICU: a randomized clinical trial. JAMA. 2014;312:514-24.

5. Bloos F, Trips E, Nierhaus A, Briegel J, Heyland DK, Jaschinski U, et al. Effect of Sodium Selenite Administration and Procalcitonin-Guided Therapy on Mortality in Patients with Severe Sepsis or Septic Shock: A Randomized Clinical Trial. JAMA Intern Med. 2016;176:1266-76.

6. Manzanares W, Lemieux M, Elke G, Langlois PL, Bloos F, Heyland DK. High-dose intravenous selenium does not improve clinical outcomes in the critically ill: a systematic review and meta-analysis. Crit Care. 2016;20:356.

7. McClave S, Taylor BE, Martindale RG, Warren MM, Johnson DR, Braunschweig C, et al. Guidelines for the Provision and Assessment of Nutrition Support Therapy in the Adult Critically Ill Patient: Society of Critical Care Medicine (SCCM) and American Society for Parenteral and Enteral Nutrition (ASPEN). J Parenter Enteral Nutr JPEN. 2016;40:159-211.

8. Weimann A, Braga M, Carli F, Higashiguchi T, Hubner M, Klek S, et al. ESPEN guideline: Clinical nutrition in surgery. Clin Nutr. 2017;36:623-50.

9. Lee SY and Ko KS. Protective effects of S-Adenosylmethionine and Its combinations with Taurine and/or Betaine against Lipopolysaccharide or Polyinosinic-polycytidylic acid-indu- ced acute hepatotoxicity. J. Cancer Prevention. 2016:21;152. DOI: 10.15430/JCP.2016.21.3.152.

10. Jeong SY, Im YN, Youm JY, Lee HK, Im SY. L-Glutamine Attenuates DSS-Induced Colitis via Induction of MAPK Phosphatase-1. Nutrients. 2018;10(3).pii: E288.

11. Nienaber A, Dolman RC, van Graan AE, Blaauw R. Prevalence of glutamine deficiency in ICU patients: A cross-sectional analytical study. Nutr J. 2016;15:73.

12. Griffiths RD, Jones C, Palmer TE. Six-month outcome of critically ill patients given glutamine-supplemented parenteral nutrition. Nutrition. 1997;13:295-302.

13. Rodas PC, Rooyackers O, Hebert C, Norberg Å, Wernerman J. Glutamine and glutathione at ICU admission in relation to outcome. Clin Sci. 2012;122:591-7.

14. Oudemans-van Straaten HM, Bosman RJ, Treskes M, van der Spoel HJ, Zandstra DF. Plasma glutamine depletion and patient outcome in acute ICU admissions. Intensive Care Med. 2001;27:84-90.

15. Hardy G, Bevan SJ, McElroy B, Palmer TE, Griffiths RD, Braidwood C. Stability of glutamine in parenteral feeding solutions. Lancet. 1993;342:186.

16. Wernerman J, Kirketeig T, Andersson B, Berthelson H, Ersson A, Friberg $\mathrm{H}$, et al. Scandinavian glutamine trial: a pragmatic multi-centre randomised clinical trial of intensive care unit patients. Acta Anaesthesiol Scand. 2011;55:812-8.

17. Palmer TE, Jones C, Griffiths RD. Effect of parenteral L-glutamine on muscle in the very severely Ill. Nutrition. 1996;12:316-20.

18. Tsujimoto T, Shimizu K, Hata N, Tagaki T, Uejima E, Ogura $\mathrm{H}$, et al. Both high and low plasma glutamine levels predict mortality in critically ill patients. Surg Today. 2017;47:1331-8.

19. Hardy G, Langlois PL, Manzanares W. Pharmaconutrition with Intravenous Selenium in Intensive Care: Back to Basics? Nutrition. 2018; 46:131-3. 